TAIWANESE JOURNAL OF MATHEMATICS

Vol. 11, No. 2, pp. 447-469, June 2007

This paper is available online at http://www.math.nthu.edu.tw/tjm/

\title{
BOUNDED-LIPSCHITZ DISTANCES ON THE STATE SPACE OF A C*-ALGEBRA
}

Frédéric Latrémolière

\begin{abstract}
Metric noncommutative geometry, initiated by Alain Connes, has known some great recent developments under the impulsion of Rieffel and the introduction of the category of compact quantum metric spaces topologized thanks to the to quantum Rieffel-Gromov-Hausdorff distance. In this paper, we undertake the first step to generalize such results and constructions to locally compact quantum metric spaces. Our present work shows how to generalize the construction of the bounded-Lipschitz metric on the state space of a $\mathrm{C}^{*}$-algebra which need not be unital, such that the topology induced by this distance on the state space is the weak* topology. In doing so we obtain some results on a state space picture of the strict topology of a $\mathrm{C}^{*}$-algebra.
\end{abstract}

\section{INTRODUCTION}

In noncommutative geometry, as suggested in $[2,3, \mathrm{Ch} .6]$, the natural way to specify a (quantum) metric on a $C^{*}$-algebra $A$ is to choose a densely-defined seminorm $L$ on $A$, which should be viewed as a generalization of a Lipschitz seminorm. Given such a seminorm $L$ and any $\alpha>0$, one can define a norm $L_{\alpha}=\max \left\{L, \alpha^{-1}\|\|.\right\}$ whose dual seminorm on $A^{\prime}$ then induces a distance $d_{L, \alpha, 1}$ on the state space $S(A)$ of $A$. The prototype for this situation is given by a locally compact separable metric space $(X, \rho)$ and by taking $L$ to be the usual Lipschitz seminorm on $A=C_{0}(X)$. The natural embedding of $X$ into the space $S\left(C_{0}(X)\right)$ of Radon probability measures over $X$, which maps any $x \in X$ to the Dirac point measure $\delta_{x}$, is also an isometry from any balls in $X$ of radius at most $\alpha$ into $\left(S\left(C_{0}(X)\right), d_{L_{\alpha}}\right)$ for any $\alpha>0$. Therefore, the family of norms $\left(L_{\alpha}\right)_{\alpha>0}$ encodes all the metric information of $X$ just as the $C^{*}$-norm encodes all the topological

Received May 12, 2005, revised September 11, 2005.

Communicated by Man-Duen Choi.

2000 Mathematics Subject Classification: Primary 46L89, 46L30.

Key words and phrases: Quantum metric spaces, Strict topology, Lip-norms, State space.

The author wishes to thank Marc Rieffel for his help and support during this research. 
information of $X$. In this context, the distances $d_{L, \alpha, 1}$ are known as the boundedLipschitz distances and have the fundamental property that any one of them induces the weak* topology on $S\left(C_{0}(X)\right)$ [6, Theorem 11.3 .3 p. 395]. A natural question, therefore, is to find out what condition on $L$ one needs to ensure that $d_{L, \alpha, 1}$ metrizes the weak* topology on $S(A)$ for any (non-Abelian) C*-algebra $A$.

When $A$ is unital, this problem was answered by Rieffel in [11, Theorems 1.8 and 1.9]: the distance $d_{L, \alpha, 1}$ induces the weak* topology on $S(A)$ if and only if the set $\{a \in A: L(a) \leq 1,\|a\| \leq \alpha\}$ is norm precompact in $A$. This generalizes the prototypical situation where $X$ is assumed to be compact, in which case the normprecompactness of any set of 1-Lipschitz uniformly bounded functions follows from Arzéla-Ascoli. Moreover, when $X$ is compact, it is of finite diameter $r$, so that as long as $L(1)=0$, the bounded-Lipschitz distance $d_{L, r, 1}$ is also the distance induced by the dual seminorm of $L$ and is known in this context as the Kantorovich distance. It is in this framework that [11, Theorems 1.8 and 1.9] is written. Following on [11], it is possible to develop a theory of Gromov-Hausdorff convergence [14] for $\mathrm{C}^{*}$-algebras with such seminorms, leading to many interesting new approximations of $\mathrm{C}^{*}$-algebras, as in $[14,13,9]$.

We undertake in this paper the first step into a generalization of the theory of compact quantum metric spaces, as developed in [11, 12] and [14], to nonunital $\mathrm{C}^{*}$-algebras. As in the unital case, this first step consists in characterizing those seminorms on (nonunital) $\mathrm{C}^{*}$-algebras whose associated bounded-Lipschitz distances induces the weak* topology on $S(A)$. We thus answer the problem:

Problem 1.1. Let $A$ be a separable $\mathrm{C}^{*}$-algebra, with or without a unit. Let $L$ be a seminorm on a dense subset $\operatorname{dom} L$ of the self-adjoint part $A^{\mathrm{sa}}$ of $A$. We extend $L$ to $A^{\text {sa }}$ by setting $L(a)=\infty$ whenever $a \notin \operatorname{dom} L$. We define the distance $d_{L}=$ $d_{L, 1,1}$ on $S(A)$ by setting $d_{L}(\varphi, \psi)=\sup \{|\varphi(a)-\psi(a)|: L(a) \leq 1,\|a\| \leq 1\}$ for all $\varphi, \psi \in S(A)$.When does the $d_{L}$-topology agree with the weak* topology on $S(A)$ ?

The choice of the constant $\alpha=1$ in Problem (1.1) can be made without loss of generality, as shown in Proposition (2.10), since we are only asking a topological, i.e. local question.

The choice of bounded-Lipschitz distances, rather than the Kantorovich distance $\kappa_{L}=d_{L, \infty, 1}$, as the natural framework when working with nonunital $\mathrm{C}^{*}$-algebras is justified by the following two simple observations about $\kappa_{L}$ when $A$ is Abelian nonunital and $L$ is the Lipschitz seminorm for some distance on the Gelfan'd spectrum $X$ of $A$. First of all, $\kappa_{L}$ is valued in $[0, \operatorname{diam} X]$ and thus can take the value $\infty$ if $X$ has infinite radius:

Example 1.2. Let $X=\mathbb{R}$ with its usual metric and denote by $L$ the usual Lipschitz seminorm on $C_{0}(\mathbb{R})$. Set $\varphi=\sum_{n=0}^{\infty} 2^{-n} \delta_{2^{2 n}} \in S\left(C_{0}(X)\right)$. For any 
set $E \subseteq \mathbb{R}$ we define $\chi_{E}$ to be the indicator function of $E$. Now, for any $n \in \mathbb{N}$ we define $f_{n}(t)=\chi_{\left[0,2^{2 n}[\right.} t+\chi_{\left[2^{2 n}, 2^{2 n+1}\right]}\left(2^{2 n+1}-t\right)$ for all $t \in \mathbb{R}$. Of course, $L\left(f_{n}\right)=1$, yet $\varphi\left(f_{n}\right) \geq 2^{n}$. Since $\delta_{0}\left(f_{n}\right)=0$ we have $\kappa_{L}\left(\varphi, \delta_{0}\right) \geq 2^{n}$ for any $n \in \mathbb{N}$ so $\kappa_{L}\left(\varphi, \delta_{0}\right)=\infty$.

Of course, one could still define a topology based on a distance taking the value $\infty$. However, when $X$ is noncompact, $\kappa_{L}$ does not in general metrize the weak* topology:

Example 1.3. We work with the notations of Example (1.2). For any $n \in$ $\mathbb{N} \backslash\{0\}$ we define $g_{n}(t)=\chi_{[0, n[} t+\chi_{[n, 2 n]}(2 n-t)$ and $\varphi_{n}=\left(\left(1-\frac{1}{n}\right) \delta_{0}+\frac{1}{n} \delta_{n}\right)$. For all $n \in \mathbb{N} \backslash\{0\}$, we have $\varphi_{n}\left(g_{n}\right)=1$ and $L\left(g_{n}\right)=1$ by construction, so this shows that $\kappa_{L}\left(\delta_{0}, \varphi_{n}\right) \geq 1$ since $\delta_{0}\left(g_{n}\right)=0$. On the other hand, for any $f \in C_{0}(\mathbb{R})$ we have $\varphi_{n}(f) \underset{n \rightarrow \infty}{\longrightarrow} \delta_{0}(f)$ so $\left(\varphi_{n}\right)_{n \in \mathbb{N}}$ weak* converge to $\delta_{0}$.

Therefore, we will focus in this paper on the bounded-Lipschitz distances, which are known to always metrize the weak* topology for locally compact spaces, and as we saw include $\kappa_{L}$ when $X$ is compact.

We adopt the point of view that Problem (1.1) is of intrinsic interest, since the state space $S(A)$ is in general a very delicate object (see for instance [1]), especially when $A$ is not unital. Thus, solving Problem (1.1) would provide a mean to bring back problems involving the weak* topology $S(A)$ to problems on the $\mathrm{C}^{*}$-algebra $A$ with a well-chosen seminorm $L$. This approach led to successful developments when $A$ is unital ([14,9] to quote a few) and we hope that further research will show similar results for the nonunital case as well, based upon our answer to Problem (1.1).

A key element in the proof of [11, Theorems 1.8 and 1.9] is that $S(A)$ is weak* compact when $A$ is unital. Of course, when $A$ is not unital, $S(A)$ is never compact for the weak* topology, as its weak* closure $Q(A)$ is the space of positive linear functionals of norm at most 1 [10]. Moreover, since $S(A)$ is not weak* open in $Q(A)$ (as $Q(A) / S(A)$ is dense), it is not even weak* locally compact. Therefore we need new topological insights to solve Problem (1.1) in a manner similar to [11, Theorems 1.8 and 1.9].

We propose a solution to Problem (1.1) which again provides a topological condition on $\mathcal{B}_{L}=\left\{a \in A^{\text {sa }}: L(a) \leq 1,\|a\| \leq 1\right\}$ which mirrors the topology of $S(A)$ : in the first part of this paper, we show that the $d_{L}$-topology is the weak* topology on $S(A)$ if and only if $\mathcal{B}_{L}$ is totally bounded for the topology wu of uniform weak convergence on weak* compact subsets of $S(A)$. Since the topology wu is still rather obscure, we construct in the second part of this paper a metrization for wu on bounded subsets and compare wu to the strict topology, which is always stronger than wu and agrees with wu on any bounded subset $B$ of $A$ if and only if the multiplication is jointly continuous for wu on $B$, which is usually not the 
case. We obtain in this process an alternative description of the strict topology on bounded subsets using $S(A)$. We then conclude with an easier answer to Problem (1.1) as a corollary.

\section{Metrizing the WeaK* Topology of the State Space of a C**-Algebra}

In this section, $A$ is a separable $\mathrm{C}^{*}$-algebra with or without a unit, and whose norm is denoted by $\|$.$\| . Its state space S(A)$ is endowed with the restriction of the weak* topology $\sigma\left(A^{\prime}, A\right)$. The self-adjoint part of $A$ is denoted by $A^{\text {sa }}$.

Definition 2.1. Let $A$ be a separable $\mathrm{C}^{*}$-algebra and $L$ be a seminorm defined on a norm-dense subset $\operatorname{dom} L$ of the set $A^{\text {sa }}$ of self-adjoint elements in $A$. We extend $L$ to $A^{\text {sa }}$ by setting $L(a)=\infty$ for all $a \notin \operatorname{dom} L$, and we set:

$$
\mathcal{B}_{L}=\{a \in \operatorname{dom} L: L(a) \leq 1,\|a\| \leq 1\} .
$$

We fix in this section a seminorm $L$ as in Definition (2.1). The following easy lemma will prove useful for defining a distance on the state space $S(A)$ of $A$ :

Lemma 2.2. Suppose that, for some $\varphi, \psi \in S(A)$ we have $\varphi(a)=\psi(a)$ for all $a \in \mathcal{B}_{L}$. Then $\varphi=\psi$.

Proof. Let $a \in \operatorname{dom} L$. Then $\left(\max \{1, L(a),\|a\|)^{-1} a \in \mathcal{B}_{L}\right.$. Hence, $\varphi(a)=$ $\psi(a)$. Now, $\operatorname{dom} L$ is norm dense in $A^{\text {sa }}$, so by continuity $\varphi=\psi$ on $A^{\text {sa }}$. Since $A^{\text {sa }}$ linearly spans $A$, we have $\varphi=\psi$ on $A$ by linearity.

Definition 2.3. Let $A$ be a separable $\mathrm{C}^{*}$-algebra and $L$ be a densely defined seminorm on the set $A^{\text {sa }}$ of self-adjoint elements of $A$. We define the boundedLipschitz distance $d_{L}$ dual to $L$ on the state space $S(A)$ of $A$ by setting, for all $\varphi, \psi \in S(A)$ :

$$
d_{L}(\varphi, \psi)=\sup \left\{|\varphi(a)-\psi(a)|: a \in \mathcal{B}_{L}\right\} .
$$

We aim at solving Problem (1.1). We start with a natural necessary condition on $L$ for $d_{L}$ to metrize $\sigma\left(A^{\prime}, A\right)$. Given a seminorm $p$ on $A$, we shall say a subset $V$ of $A$ is totally bounded for $p$ when for any $\varepsilon>0$ there exists a finite subset $F_{\varepsilon}$ of $A$ such that $V \subseteq\{a \in A: p(a) \leq \varepsilon\}+F_{\varepsilon}$.

Lemma 2.4. If the $d_{L}$-topology is the weak ${ }^{*}$ topology on $S(A)$ then, for all weak* compact subsets $K$ of $S(A)$, the set $\mathcal{B}_{L}$ is totally bounded for the seminorm $p_{K}$ on $A$ defined for all $a \in A$ by $p_{K}(a)=\sup _{\varphi \in K}|\varphi(a)|$. 
Proof. Assume that $d_{L}$ metrizes the topology $\sigma\left(A^{\prime}, A\right)$ on $S(A)$. Let $K$ be a weak* compact subset of $S(A)$.For any $a \in A$ we denote by $\widehat{a}$ the affine function $\widehat{a}: \varphi \in K \longmapsto \varphi(a)$. The map $\widehat{a}$ is continuous on $K$ by definition of the weak* topology. Let $C(K)$ be the Banach space of continuous functions on $K$ endowed with the supremum norm. The map $\xi_{K}: a \longmapsto \widehat{a} \in C(K)$ is an isometry for $p_{K}$ by definition. Since $p_{K} \leq\|$.$\| , the map \xi_{K}$ is also continuous from $A$ into $C(K)$. We have, by definition of $d_{L}$, for all $\varphi, \psi \in K$ and for all $a \in \mathcal{B}_{L}$ :

$$
|\widehat{a}(\varphi)-\widehat{a}(\psi)|=|\varphi(a)-\psi(a)| \leq d_{L}(\varphi, \psi) .
$$

Now, $d_{L}$ metrizes $S(A)$ for the weak* topology, and so in particular the topology on $K$ obtained from $d_{L}$ is the restriction of $\sigma\left(A^{\prime}, A\right)$ on $K$. Therefore, for all $a \in \mathcal{B}_{L}$, the functions $\widehat{a}$ are equicontinuous over the weak* compact $K$. They also are, by definition, valued in the common compact $[-1,1]$. By Arzela-Ascoli, $\mathcal{B}_{L}$ is totally bounded for the seminorm $p_{K}$.

This proof is rather classic, and can be found in [11, Theorem 1.8] when $A$ is a unital $\mathrm{C}^{*}$-algebra, in which case the state space $S(A)$ is itself weak* compact and the functional representation $a \in A^{\mathrm{sa}} \longmapsto \widehat{a} \in \operatorname{Af}(S(A))$ is a surjective linear order-preserving isometry (see [10, sec. 3.10, pp. 69-73]Pedersen79) known as the Kadison representation [8], where $\operatorname{Af}(S(A))$ is the space of continuous affine functions on the convex $S(A)$.

The proof of Lemma (2.4) now suggests that we define the following topology, in search of the proof of a sufficient condition:

Definition 2.5. Let $A$ be a separable $\mathrm{C}^{*}$-algebra. We denote by $\mathfrak{S}$ the set of all weak* compact subsets of the state space $S(A)$ of $A$. For any $K \in \mathfrak{S}$ we define the seminorm $p_{K}$ on $A$ by:

$$
p_{K}(a)=\sup _{\varphi \in K}|\varphi(a)| \text { for all } a \in A .
$$

The locally convex topology on $A$ generated by the family $\left(p_{K}\right)_{K \in \mathfrak{S}}$ of seminorms is called the weakly-uniform topology on $A$ and is denoted by wu.

Thus, the topology wu is the topology of uniform weak convergence on weak* compact subsets of $S(A)$. Note that a subset of $S(A)$ is weak* compact in $A^{\prime}$ if and only if it is weak* compact in $S(A)$.

We will study the main properties of wu in the next section. This topology is the natural setting not only for the necessary condition of Lemma (2.4), but also for the sufficient condition in the following theorem which is the first step toward answering Problem (1.1):

Theorem 2.6. Let $L$ be a seminorm defined on a norm-dense subset $\operatorname{dom} L$ of the set $A^{\mathrm{sa}}$ of self-adjoint elements of a separable $C^{*}$-algebra $A$. Let $\mathcal{B}_{L}=$ 
$\{a \in \operatorname{dom} L: L(a) \leq 1,\|a\| \leq 1\}$. The bounded-Lipschitz distance $d_{L}$ dual to $L$ metrizes the weak* topology on the state space $S(A)$ of $A$ if, and only if, the set $\mathcal{B}_{L}$ is totally bounded for the weak-uniform topology wu.

Proof. We established most of the necessary conditions in Lemma (2.4). Let us thus assume that $d_{L}$ metrizes the weak* topology on $S(A)$. Let $V$ be any open neighborhood of 0 for the wu topology. There exists a set $K \in \mathfrak{S}$ and $\varepsilon>0$ such that $\left\{a: p_{K}(a)<\varepsilon\right\} \subseteq V$. By Lemma (2.4), there exists a finite subset $F$ of $A$ such that $\mathcal{B}_{L} \subseteq\left\{a: p_{K}(a)<\varepsilon\right\}+F \subseteq V+F$. Hence, $\mathcal{B}_{L}$ is wu-totally bounded.

Conversely, assume that $\mathcal{B}_{L}$ is wu-totally bounded. Let $\left(\varphi_{n}\right)_{n \in \mathbb{N}}$ be a sequence in $S(A)$ converging in $\sigma\left(A^{\prime}, A\right)$ to a state $\varphi_{\infty}$. Since $K=\left\{\varphi_{n}: n \in \mathbb{N} \cup\{\infty\}\right\} \in$ $\mathfrak{S}$, by assumption $\mathcal{B}_{L}$ is totally bounded for $p_{K}$. Let $\varepsilon>0$ and let $V$ be the wuopen set $\left\{a \in A: p_{K}(a)<\frac{\varepsilon}{3}\right\}$. There exists a finite subset $F=\left\{a_{1}, \ldots, a_{\# F}\right\}$ of $A$ such that $\mathcal{B}_{L} \subseteq V+F$. Let $N$ be chosen such that for all $n \geq N$, for all $i=0, \ldots, \# F$, we have $\left|\varphi_{n}\left(a_{i}\right)-\varphi_{\infty}\left(a_{i}\right)\right| \leq \frac{1}{3} \varepsilon$. Let $a \in \mathcal{B}_{L}$. There exists $a_{i} \in F$ such that $a-a_{i} \in V$. Therefore, for all $n \geq N$ :

$$
\begin{aligned}
\left|\varphi_{n}(a)-\varphi_{\infty}(a)\right| & \leq\left|\varphi_{n}\left(a-a_{i}\right)\right|+\left|\varphi_{\infty}\left(a-a_{i}\right)\right|+\left|\varphi_{n}\left(a_{i}\right)-\varphi_{\infty}\left(a_{i}\right)\right| \\
& \leq \frac{2}{3} \varepsilon+\frac{1}{3} \varepsilon=\varepsilon .
\end{aligned}
$$

Hence $d_{L}\left(\varphi_{n}, \varphi_{\infty}\right) \leq \varepsilon$. Since $A$ is separable, the topology $\sigma\left(A^{\prime}, A\right)$ is metrizable on $S(A)$ by Lemma (2.11), so this concludes our proof.

Remark 2.7. Theorem (2.6) can be proven when $A$ is any topological space and $S(A)$ is any subset of $A^{\prime}$ such that the weak* topology on $S(A)$ is metrizable. We will explore the relation between wu and the algebraic structure on $A$ in later sections.

Theorem (2.6) suggest the following definition:

Definition 2.8. A seminorm $L$ defined on a dense subset $\operatorname{dom} L$ of the set of self-adjoint elements of a separable $\mathrm{C}^{*}$-algebra $A$ and such that its dual boundedLipschitz distance $d_{L}$ induces the restriction of the weak* topology on the state space $S(A)$ of $A$ is called a quasi-Lip-norm.

The terminology of quasi-Lip-norm is explained by the following simple result:

Corollary 2.9. Let $A$ be a unital separable $C^{*}$-algebra and $L$ is a quasi-Lipnorm on A. Then L is a Lip-norm (as defined in [11, Theorem 1.9]) if and only if the following two conditions hold:

(1) $\{a \in \operatorname{dom} L: L(a)=0\}=\mathbb{R} 1$,

(2) $\sup \left\{d_{L}(\varphi, \psi): \varphi, \psi \in S(A)\right\}<\infty$. 
Proof. Apply Theorem [11, Theorem 1.9] with Theorem (2.6).

On the other hand, whether $A$ is unital or not, the diameter of $S(A)$ for $d_{L}$ is always 2. This may seem arbitrary (though see [16, p. 2 and sec 1.7, pp. 2427]), however as far as the topology is concerned, we could have chosen different bounds on $L$ and $\|$.$\| to define a distance on S(A)$ without much consequence for our purposes:

Proposition 2.10. Let $A$ be a separable $C^{*}$-algebra and let $L$ a seminorm defined on a dense subset $\operatorname{dom} L$ of the self-adjoint part of $A$. Let $\alpha, \beta>0$. The $(\alpha, \beta)$-bounded-Lipschitz metric $d_{L, \alpha, \beta}$ defined on the state space $S(A)$ of $A$ by:

$$
d_{L, \alpha, \beta}(\varphi, \psi)=\sup \{|\varphi(a)-\psi(a)|: a \in \operatorname{dom} L, L(a) \leq \beta,\|a\| \leq \alpha\}
$$

is equivalent to the bounded-Lipschitz metric $d_{L}$. Consequently, $d_{L, \alpha, \beta}$ metrizes the weak* topology on $S(A)$ if and only if $L$ is a quasi-Lip-norm.

Proof. Simply remark that if $\mathcal{B}_{L}^{\prime}=\{a \in \operatorname{dom} L: L(a) \leq \beta,\|a\| \leq \alpha\}$ then $(\max \{\alpha, \beta\})^{-1} \mathcal{B}_{L}^{\prime} \subseteq \mathcal{B}_{L} \subseteq(\min \{\alpha, \beta\})^{-1} \mathcal{B}_{L}^{\prime}$.

Informally, the family of metrics $\left(d_{L, \alpha, 1}\right)_{\alpha \in \mathbb{R}}$ describes the global metric geometry of $S(A)$ while any metric of the family only describes the local metric properties of $S(A)$ (in particular one can only recover $L$ from the whole family $\left(d_{L, \alpha, 1}\right)_{\alpha \in \mathbb{R}}$ ).

To conclude this section, we propose a simple criterion on a seminorm $L$ for the distance $d_{L}$ to be complete:

Proposition 2.11. The state space $S(A)$ of a (separable) $C^{*}$-algebra $A$ is a $G_{\delta}$ in the weak ${ }^{*}$ compact space $Q(A)$ of positive linear functionals of norm at most 1 , so $S(A)$ is a Polish space: it is metrizable for the weak* topology with a complete metric. Let $L$ be a quasi-Lip-norm on $A$, and let $d_{L}$ be the bounded-Lipschitz distance induced by $L$ on $S(A)$. Then $\left(S(A), d_{L}\right)$ is a complete path-metric space if there exists an approximate unit $\left(e_{n}\right)_{n \in \mathbb{N}}$ in $A$ such that for all $n \in \mathbb{N}$ we have $L\left(e_{n}\right) \leq 1$ and $\left\|e_{n}\right\| \leq 1$.

Proof. Simply observe $S(A)=\bigcap_{n=1}^{\infty}\left(Q(A) \backslash \frac{n-1}{n} Q(A)\right)$ where $\frac{n-1}{n} Q(A)$ is trivially a weak* closed subset of $Q(A)$ to conclude that $S(A)$ is a $G_{\delta}$. We have not used here that $A$ is separable.

Let $\left(e_{n}\right)_{n \in \mathbb{N}}$ be an approximate unit of $A$ contained in $\mathcal{B}_{L}$. Let $\left(\varphi_{n}\right)_{n \in \mathbb{N}}$ be a Cauchy sequence of states of $A$ for the bounded Lipschitz distance $d_{L}$. By linearity, $\left(\varphi_{n}(a)\right)_{n \in \mathbb{N}}$ is a Cauchy sequence for all $a \in \operatorname{dom} L$. Since $\operatorname{dom} L$ is norm-dense in $A$, and the sequence $\left(\varphi_{n}\right)_{n \in \mathbb{N}}$ is norm-bounded by assumption (it is a sequence 
of states), we conclude that for all $a \in A$ the sequence $\left(\varphi_{n}(a)\right)_{n \in \mathbb{N}}$ is a Cauchy sequence in $\mathbb{C}$, so it converges to some number $\mu(a)$. It is immediate that $\mu$, as the pointwise limit of continuous linear functionals on a Banach space, is itself a continuous linear functional. It is also immediate that $\mu$ is positive, and hence of norm $\lim _{n \rightarrow \infty} \mu\left(e_{n}\right)$. On the other hand, $\left(\varphi_{n}\right)_{n \in \mathbb{N}}$ converges uniformly to $\mu$ on $\mathcal{B}_{L}$ by definition of $d_{L}$ (extended to $A^{\prime}$ ), so we conclude $\lim _{n \rightarrow \infty} \mu\left(e_{n}\right)=$ $\lim _{k \rightarrow \infty} \lim _{n \rightarrow \infty} \varphi_{k}\left(e_{n}\right)=1$. Hence $\mu \in S(A)$ and $\left(\varphi_{n}\right)_{n \in \mathbb{N}}$ converges to $\mu$ in $\left(S(A), d_{L}\right)$.

Now it is easy to check that, if $\varphi, \psi \in S(A)$, then $\eta=\frac{1}{2}(\varphi+\psi) \in S(A)$ as $S(A)$ is convex, and moreover $d_{L}(\eta, \psi)=\frac{1}{2} d_{L}(\varphi, \psi)$. Since $S(A)$ is complete for $d_{L}$, we deduce from [7, Theorem 1.8 p. 7] that $\left(S(A), d_{L}\right)$ is a path metric space.

Now, Theorem (2.6) relies upon a somewhat mysterious description of wu. Our goal is now to reformulate Theorem (2.6) by using a more explicit description of wu on bounded subsets of $A$. To obtain this reformulation, we shall compare wu and the strict topology on $A$.

\section{The Strict Topology and the Weakly Uniform Topology}

We let $A$ be a separable $\mathrm{C}^{*}$-algebra whose norm is denoted by $\|$.$\| and whose$ state space $S(A)$ is endowed with the weak* topology. In this section, we wish to compare our topology wu to the strict topology. This is equivalent to investigate the joint continuity of the multiplication of $A$ for wu on bounded sets, where a bounded set will always mean bounded for the norm topology (we will justify this abuse of language soon). Our approach consists in metrizing wu on bounded subsets of $A$. Our main result is then that the strict topology and wu agree on bounded subsets if and only the multiplication is jointly continuous for wu on bounded subsets, which is not true in general (but is true in the Abelian and unital case).

\subsection{First Elements of Comparison}

We start very simply with the trivial remark:

Proposition 3.1. If $A$ is a unital $C^{*}$-algebra, then the weakly-uniform topology is the norm topology on $A$.

Proof. Since $A$ is unital, $S(A) \in \mathfrak{S}$. Now $p_{S(A)}$ is equivalent to $\|$.$\| , and the$ seminorms $p_{K}$ for $K \in \mathfrak{S}$ are all dominated by $p_{S(A)}$, so wu is the norm topology. 
In the sequel, we will not assume that $A$ is unital. All of the results which follow are valid in the unital case, but they are trivial because of Proposition (3.1). The reader may thus as well assume $A$ is not unital, and thus that $S(A)$ is not $\sigma\left(A^{\prime}, A\right)$ compact.

The reader is probably already convinced that:

Lemma 3.2. On any separable $C^{*}$-algebra $A$, the weakly-uniform topology wu is stronger than the weak topology $\sigma\left(A, A^{\prime}\right)$ on $A$, and in particular wu is Hausdorff.

Proof. Since any $\psi \in A^{\prime}$ is a linear combination of four states by [10, Secs. 3.1 and 3.2 pp. 41-46], the smallest topology which makes all states continuous is the weak* topology. Since $\{\varphi\} \in \mathfrak{S}$ for any $\varphi \in S(A)$, all the states of $A$ are continuous for wu, hence our lemma.

In particular, a subset of $A$ is topologically bounded for any of the wu, weak, strict or norm topologies if and only if it is bounded for the norm topology by [15, Theorem 3.18, p. 70]. This justifies that we shall simply call such a set bounded without reference to any topology.

We now offer a first comparison between wu and the strict topology. This proposition differs from later comparisons by taking place on the whole of $A$ rather than only on bounded subsets of $A$.

Proposition 3.3. The weakly-uniform topology is weaker than the strict topology on any separable $C^{*}$-algebra $A$.

Proof. For all $a, b \in A$ and $\mu \in A^{\prime}$ we define $(a \cdot \mu)(b)=\mu(a b)$. The exterior composition law gives $A^{\prime}$ a Banach $A$-module structure. Let $K$ be a compact subset of $S(A)$ for the weak* topology. Let $\left(e_{n}\right)_{n \in \mathbb{N}}$ be any approximate unit of $A$ which is also an increasing Abelian sequence of positive elements of norm 1. Let us embed $A$ into its smallest unitalization $\widetilde{A}$ of unit 1 . We then have, by Cauchy-Schwarz:

$$
\begin{aligned}
\left\|e_{n} \cdot \varphi-\varphi\right\|^{2} & =\sup _{a \in A,\|a\| \leq 1}\left|e_{n} \cdot \varphi(a)-\varphi(a)\right|^{2}=\sup _{a \in A,\|a\| \leq 1} \varphi\left(a\left(e_{n}-1\right)\right)^{2} \\
& \leq \sup _{a \in A,\|a\| \leq 1} \varphi\left(a^{*} a\right) \varphi\left(\left(e_{n}-1\right)^{2}\right) \leq \varphi\left(\left(e_{n}-1\right)^{2}\right) .
\end{aligned}
$$

Since $\left(e_{n}\right)_{n \in \mathbb{N}}$ is Abelian, the sequence $\left(\left(1-e_{n}\right)^{2}\right)_{n \in \mathbb{N}}$ is decreasing. As in Lemma (2.4), for all $a \in A$ we define $\widehat{a}: \varphi \in K \mapsto \varphi(a)$. Then $\left(\widehat{\left(1-e_{n}\right)^{2}}\right)_{n \in \mathbb{N}}$ is a decreasing sequence of continuous functions converging pointwise to 0 in the compact $K$, hence converges uniformly by Dini's theorem, so $\sup _{\varphi \in K}\left\|e_{n} \cdot \varphi-\varphi\right\| \underset{n \rightarrow \infty}{\longrightarrow} 0$. 
The set $K$ is bounded, so by [5, Theorem 17.1, p. 114], a corollary the Cohen Factorization Theorem, there exists $c \in A$ and some bounded subset $Y$ of $A^{\prime}$ such that $K=c \cdot Y$. Therefore, for all $a \in A$ :

$$
p_{K}(a)=\sup _{\varphi \in K}|\varphi(a)|=\sup _{\mu \in Y}|\mu(c a)| \leq\left(\sup _{\mu \in Y}\|\mu\|\right)\|c a\| .
$$

Since $Y$ is bounded, the seminorm $p_{K}$ is strictly continuous.

\subsection{Inductive Topologies}

We construct the bridge between the strict topology and the topology wu in this section. This connection is established by introducing a family of topologies which, at least on bounded subsets of $A$, will all agree with wu, but are also very close in definition with the strict topology. These topologies will be defined using sequences which, to ease later estimates, we will allow to be in the enveloping Von Neumann algebra $A^{\prime \prime}$ of $A$ (so that we can always choose sequences of projections). Since the canonical map $A \rightarrow A^{\prime \prime}$ is a *-monomorphism and hence an isometry, we still denote by $\|$.$\| the norm on the enveloping Von Neumann algebra A^{\prime \prime}$ of $A$. Any state of $A$ is extended uniquely to a normal state of $A^{\prime \prime}$. We define:

Definition 3.4. Let $A$ be a separable $\mathrm{C}^{*}$-algebra whose bidual is denoted by $A^{\prime \prime}$. Let $\left(e_{n}\right)_{n \in \mathbb{N}}$ be a sequence in $A^{\prime \prime}$. We assume moreover that $\left(e_{n}\right)_{n \in \mathbb{N}}$ satisfies the following condition:

\section{Condition 3.5.}

$$
\forall \alpha \in \mathbb{N} \backslash\{0\} \quad \forall K \in \mathfrak{S} \quad \lim _{n \rightarrow \infty} p_{K}\left(\left(1-e_{n}\right)^{\alpha}\right)=0 .
$$

We define on $A$ the seminorms $I_{e_{n}}: a \longmapsto\left\|e_{n} a e_{n}\right\|$ for all $n \in \mathbb{N}$. The locally convex topology on $A$ generated by the seminorms $\left(I_{e_{n}}\right)_{n \in \mathbb{N}}$ is called the inductive topology along $\left(e_{n}\right)_{n \in \mathbb{N}}$ and we will denote it by ind $\left(e_{n}\right)_{n \in \mathbb{N}}$.

For Definition (3.4) to be nonvacuous, we construct sequences $\left(e_{n}\right)_{n \in \mathbb{N}}$ such that Condition (3.5) holds. Since $A$ is separable, it contains a strictly positive element, so we can define:

Definition 3.6. Let $A$ be a separable $\mathrm{C}^{*}$-algebra. Let $h \in A$ be a strictly positive element such that $\|h\|=1$ and whose spectrum in $A$ is denoted by $\sigma(h)$. Let $\left(f_{n}\right)_{n \in \mathbb{N}}$ be a sequence of nondecreasing functions on $\sigma(h) \cup\{0\} \subseteq[0,1]$ such that for all $n \in \mathbb{N}$ we have $f_{n}(0)=0$ and $f_{n}(1)=1$. We also assume that $\left(f_{n}\right)_{n \in \mathbb{N}}$ converges pointwise to 1 on $\sigma(h) \backslash\{0\}$. The sequence $\left(f_{n}(h)\right)_{n \in \mathbb{N}}$ of elements in 
the enveloping Von Neumann algebra $A^{\prime \prime}$ of $A$ will be called a ( $h$-)pseudo-spectral (ps-) approximate unit of $A$.

Remark 3.7. To distinguish ps-approximate units from the usual approximate units in $A$, we shall call the latter continuous approximate units, and adopt the convention that all continuous approximate units are positive and of norm uniformly bounded by 1 .

Since any monotone function is a Borel function, ps-approximate units are welldefined by the Borel functional calculus in $W^{*}(h)$ and are Abelian sequences of positive elements of norm 1 in $A^{\prime \prime}$. We will prove later in Proposition (3.16) that ps-approximate units satisfy a similar approximation property in wu to the defining approximation property of continuous approximate units in the strict topology, yet for now we do not need this fact. We now prove that there are many examples of sequences verifying Condition (3.5):

Lemma 3.8. Let $\left(e_{n}\right)_{n \in \mathbb{N}}$ be an Abelian nondecreasing continuous approximate unit of $A$ of positive elements of norm at most 1 , or a ps-approximate unit of $A$. Let 1 denote the identity of $A^{\prime \prime}$. Let $K \in \mathfrak{S}$. We have for all $\alpha \in \mathbb{N} \backslash\{0\}$ :

$$
\lim _{n \rightarrow \infty} \sup _{\varphi \in K}\left|\varphi\left(\left(1-e_{n}\right)^{\alpha}\right)\right|=0 .
$$

Proof. We denote by $Q(A)$ the space of positive linear functionals of norm at most 1 on $A$, and we endow $Q(A)$ with the weak* topology. The space $Q(A)$ is often known as the quasi-state space of $A$ [10]. Let $a \in A^{\prime \prime}$ be self-adjoint. The function $\widehat{a}$ defined on $Q(A)$ by $\varphi \mapsto \varphi(a)$ is an affine Borel map vanishing at 0 . By $\left[10\right.$, Theorem 3.10.3, p. 70], the map $\xi: a \in A^{\prime \prime}$ sa $\mapsto \widehat{a}$ is a linear order-preserving isometry from $A^{\prime \prime s a}$ into the space $B_{0}(Q(A))$ of real Borel affine functions on $Q(A)$ vanishing at 0 , and the image of $A^{\text {sa }}$ by $\xi$ is the subspace $A_{0}(Q(A))$ of $B_{0}(Q(A))$ of real continuous affine functions on $Q(A)$ vanishing at 0 .

We first recall the standard argument for Abelian continuous approximate units. Let $K \in \mathfrak{S}$ and let $\alpha \in \mathbb{N} \backslash\{0\}$. Since $\left(e_{n}\right)_{n \in \mathbb{N}}$ is Abelian, the sequence $\left(\left(1-e_{n}\right)^{\alpha}\right)_{n \in \mathbb{N}}$ is also an Abelian nondecreasing continuous approximate unit. The sequence $\left(\left(\widehat{\left(-e_{n}\right)^{\alpha}}\right)_{n \in \mathbb{N}}\right.$ is a nonincreasing sequence of continuous affine functions on the compact $K$ whose limit is the continuous function 0 . Therefore, by Dini's theorem, $\left(\overline{\left(1-e_{n}\right)^{\alpha}}\right)_{n \in \mathbb{N}}$ converges uniformly on $K$ to 0 , which proves (3.1) by definition. This part of the proof did not require that $\left(e_{n}\right)_{n \in \mathbb{N}}$ is Abelian when $\alpha=1$.

We now turn to the case when $\left(e_{n}\right)_{n \in \mathbb{N}}$ is a ps-approximate unit. Let $K \in \mathfrak{S}$. Let $h$ be a strictly positive element of norm 1 such that for all $n \in \mathbb{N}$, there 
exists a nondecreasing function $f_{n}$ on $\sigma(h) \cup\{0\}$ such that $e_{n}=f_{n}(h)$ in $A^{\prime \prime}$. For any $\mu \in A^{\prime}$, we define $\pi(\mu)$ to be its restriction to $C^{*}(h)$. By construction, $\pi: A^{\prime} \rightarrow C^{*}(h)^{\prime}$ is a positive linear surjection of norm 1. Moreover, since $C^{*}(h)$ contains a continuous approximate unit since $h$ is strictly positive (e.g. $\left(1-(1-h)^{n}\right)_{n \in \mathbb{N}}$ ), we have $\pi(\varphi) \in S\left(C^{*}(h)\right)$ for any state $\varphi \in S(A)$. Let $K^{\prime}=\pi(K) \subseteq S\left(C^{*}(h)\right)$. As a norm-continuous linear function, $\pi$ is also a continuous map between $A^{\prime}$ and $C^{*}(h)^{\prime}$ endowed with their respective weak* topologies, so $K^{\prime}$ is weak* compact in $S\left(C^{*}(h)\right)$. Since for all $\alpha \in \mathbb{N} \backslash\{0\}$ and all $n \in \mathbb{N}$, we have $\sup _{\varphi \in K}\left|\varphi\left(\left(1-e_{n}\right)^{\alpha}\right)\right|=\sup _{\varphi \in K^{\prime}}\left|\varphi\left(\left(1-e_{n}\right)^{\alpha}\right)\right|$, we can now work solely in the Abelian $C^{*}$-algebra $C^{*}(h)$. Since $K^{\prime}$ is weak* compact, and $\sigma(h) \backslash\{0\}$ is a Polish locally compact space, $K^{\prime}$ is uniformly tight. Let $\varepsilon>0$. There exists a compact subset $k_{\varepsilon}$ of $\sigma(h) \backslash\{0\}$ such that $\sup \left\{\varphi\left(\sigma(h) \backslash k_{\varepsilon}\right): \varphi \in K^{\prime}\right\} \leq \varepsilon$, where we identify Radon integrals with their Radon measures. Hence, for any $\alpha \in \mathbb{N} \backslash\{0\}, n \in \mathbb{N}$ we have:

$$
\sup _{\varphi \in K^{\prime}}\left|\varphi\left(\left(1-f_{n}\right)^{\alpha}\right)\right| \leq \max \left\{\varepsilon^{\alpha}, \sup _{\varphi \in K^{\prime}}\left(\int_{k_{\varepsilon}}\left(1-f_{n}\right)^{\alpha} d \varphi\right)\right\} .
$$

The sequence of nonincreasing functions $\left(1-f_{n}\right)_{n \in \mathbb{N}}^{\alpha}$ converges pointwise on the compact subset $k_{\varepsilon}$ to the continuous function 0 by assumption. Let $m=\min k_{\varepsilon}$ and $M=\max k_{\varepsilon}$. Then we have, for all $n \in \mathbb{N}$ and all $x \in k_{\varepsilon}$ :

$$
\left(1-f_{n}\right)^{\alpha}(M) \leq\left(1-f_{n}\right)^{\alpha}(x) \leq\left(1-f_{n}\right)^{\alpha}(m)
$$

so, since $\left(1-f_{n}(m)\right)_{n \in \mathbb{N}}^{\alpha}$ and $\left(1-f_{n}(M)\right)_{n \in \mathbb{N}}^{\alpha}$ converge to 0 we deduce that $(1-$ $\left.f_{n}\right)_{n \in \mathbb{N}}^{\alpha}$ converges uniformly on $k_{\varepsilon}$ to 0 for any $\alpha \in \mathbb{N} \backslash\{0\}$. Therefore there exists $N \in \mathbb{N}$ such that for all $n \geq N$ we have $\sup _{k_{\varepsilon}}\left(1-f_{n}\right)^{\alpha} \leq \varepsilon^{\alpha}$. So, by (3.2), we have $\sup _{\varphi \in K^{\prime}}\left|\varphi\left(\left(1-e_{n}\right)^{\alpha}\right)\right| \leq \varepsilon^{a}$. This concludes our proof since $\varepsilon>0$ is arbitrary.

The main motivation for Definition (3.4) is the following proposition:

Proposition 3.9. Let $A$ be a separable $C^{*}$-algebra, and let $A^{\prime \prime}$ be its bidual, which is the enveloping Von Neumann algebra of $A$. Let $\left(e_{n}\right)_{n \in \mathbb{N}} \in A^{\prime \prime}$ be a sequence satisfying Condition (3.5). A bounded net converges for the weaklyuniform topology wu if it converges in the inductive topology ind $\left(e_{n}\right)_{n \in \mathbb{N}}$. In other words, ind $\left(e_{n}\right)_{n \in \mathbb{N}}$ is finer than wu on bounded subsets of $A$.

Proof. Since any $a \in A$ can be written as a linear combination of the two self- adjoint elements $\frac{1}{2}\left(a+a^{*}\right)$ and $\frac{1}{2 i}\left(a-a^{*}\right)$, and since both topologies make the addition, scalar multiplication and the involution of $A$ continuous, we will restrict ourselves to nets of self-adjoint elements of norm bounded by 1 and converging to 0 . 
Let now $\left(a_{\lambda}\right)_{\lambda \in \Lambda}$ be a net in $A^{\text {sa }}$ converging to $0 \in A$ for ind $\left(e_{n}\right)_{n \in \mathbb{N}}$ and uniformly bounded in norm by 1 . Let $K \in \mathfrak{S}$. Let $\varepsilon>0$. By assumption, $\left(e_{n}\right)_{n \in \mathbb{N}}$ satisfies Condition (3.5), so there exists $N \in \mathbb{N}$ such that, for all $\varphi \in K$ and all $n \geq N$ we have $\varphi\left(\left(1-e_{n}\right)^{2}\right) \leq \varepsilon^{2}$. Choose $n \geq N$ and $\varphi \in K$. Now:

$$
\begin{aligned}
\varphi\left(a_{\lambda}\right) & =\varphi\left(e_{n} a_{\lambda} e_{n}\right)+\varphi\left(\left(1-e_{n}\right) a_{\lambda} e_{n}\right)+ \\
& +\varphi\left(e_{n} a_{\lambda}\left(1-e_{n}\right)\right)+\varphi\left(\left(1-e_{n}\right) a_{\lambda}\left(1-e_{n}\right)\right) .
\end{aligned}
$$

By the Cauchy-Schwarz inequality:

$$
\begin{aligned}
\left|\varphi\left(\left(1-e_{n}\right) a_{\lambda} e_{n}\right)\right| & \leq \sqrt[2]{\varphi\left(\left(1-e_{n}\right)^{2}\right)} \sqrt[2]{\varphi\left(e_{n} a_{\lambda}^{*} a_{\lambda} e_{n}\right)} \\
& \leq \sqrt[2]{\varphi\left(\left(1-e_{n}\right)^{2}\right)} \leq \varepsilon
\end{aligned}
$$

Similarly $\left|\varphi\left(e_{n} a_{\lambda}\left(1-e_{n}\right)\right)\right| \leq \varepsilon$ and $\left|\varphi\left(\left(1-e_{n}\right) a_{\lambda}\left(1-e_{n}\right)\right)\right| \leq \varepsilon$. Thus $\left|\varphi\left(a_{\lambda}\right)\right| \leq$ $\left|\varphi\left(e_{n} a_{\lambda} e_{n}\right)\right|+3 \varepsilon$, so:

$$
p_{K}\left(a_{\lambda}\right) \leq \sup _{\varphi \in K}\left|\varphi\left(e_{n} a_{\lambda} e_{n}\right)\right|+3 \varepsilon \leq I_{e_{n}}\left(a_{\lambda}\right)+3 \varepsilon .
$$

Since the net $\left(a_{\lambda}\right)_{\lambda \in \Lambda}$ converges to 0 for ind $\left(e_{n}\right)_{n \in \mathbb{N}}$, there exists $\lambda_{0} \in \Lambda$ so that, for $\lambda \in \Lambda$ such that $\lambda \geq \lambda_{0}$ we have $I_{e_{n}}\left(a_{\lambda}\right) \leq \varepsilon$ and therefore $p_{K}\left(a_{\lambda}\right) \leq 4 \varepsilon$. So the net $\left(a_{\lambda}\right)_{\lambda \in \Lambda}$ converges to 0 for the wu topology.

As a corollary of Proposition (3.9), we have as claimed a first element of comparison between the strict topology and wu:

Corollary 3.10. Let $A$ be a separable $C^{*}$-algebra. The weakly-uniform topology wu on any bounded subset $B$ of $A$ is weaker than the strict topology on $B$.

Proof. Let $\left(a_{\lambda}\right)_{\lambda \in \Lambda}$ be a bounded net in $A$, converging strictly to 0 . Let $\left(e_{n}\right)_{n \in \mathbb{N}}$ satisfy Condition (3.5). Since the multiplication is (jointly) continuous on $B$ for the strict topology, the bounded nets $\left(e_{n} a_{\lambda} e_{n}\right)_{\lambda \in \Lambda}$ strictly converge to 0 for all $n \in \mathbb{N}$. By definition of the strict topology we have $\lim _{\lambda \in \Lambda}\left\|e_{n} a_{\lambda} e_{n}\right\|=0$ for all $n \in \mathbb{N}$. Thus $\left(a_{\lambda}\right)_{\lambda \in \Lambda}$ converges to 0 in wu by Proposition (3.9).

Thanks to Proposition (3.9), it is also possible to show that the multiplication map is not jointly continuous on bounded sets for the wu topology on the algebra $\mathcal{K}$ of compact operators on the separable Hilbert space $\mathcal{H}$.

Proposition 3.11. In general, the multiplication is not jointly continuous on bounded sets for the weakly-uniform topology wu. A counter-example is given in the unit ball of the $C^{*}$-algebra $\mathcal{K}$. 
Proof. Let $\left(\zeta_{n}\right)_{n \in \mathbb{N}}$ be an orthonormal basis for $\mathcal{H}$. Denote the inner product of $\mathcal{H}$ by $\langle.,$.$\rangle . For each n \in \mathbb{N}$ we define the compact operator $S_{n}: \xi \in \mathcal{H} \longmapsto$ $\left\langle\xi, \zeta_{0}\right\rangle \zeta_{n}$. It is easy to see that, for all $n \in \mathbb{N}$, we have $S_{n}^{*}(\xi)=\left\langle\xi, \zeta_{n}\right\rangle \zeta_{0}$ and thus $S_{n}^{*} S_{n}(\xi)=\left\langle\xi, \zeta_{0}\right\rangle \zeta_{0}$. Of course, $\left(S_{n}\right)_{n \in \mathbb{N}},\left(S_{n}^{*}\right)_{n \in \mathbb{N}}$ and $\left(S_{n}^{*} S_{n}\right)_{n \in \mathbb{N}}$ are bounded sequences. For all $n \in \mathbb{N}$, we let $P_{n}$ be the projection in $\mathcal{H}$ on the span of $\left\{\zeta_{0}, \ldots, \zeta_{n}\right\}$. The sequence $\left(P_{n}\right)_{n \in \mathbb{N}}$ is an (continuous) increasing positive Abelian approximate unit for $\mathcal{K}$ and of course $\left\|P_{n}\right\|=1$ for all $n \in \mathbb{N}$. In addition, we can check easily that, for all $n \in \mathbb{N}$ and for all $k>n$ we have $P_{n} S_{k} P_{n}=0$ and $P_{n} S_{k}^{*} P_{n}=0$. Hence, $\left(S_{n}\right)_{n \in \mathbb{N}}$ and $\left(S_{n}^{*}\right)_{n \in \mathbb{N}}$ converge to 0 in ind $\left(P_{n}\right)_{n \in \mathbb{N}}$, and therefore in the wu topology by Proposition (3.9). Yet $\left(S_{n}^{*} S_{n}\right)_{n \in \mathbb{N}}$ is constant and nonzero.

\subsection{Algebraic Description of the Weakly-Uniform Topology}

We now use a specific type of ps-approximate units to give our first algebraic description of the topology wu:

Definition 3.12. Let $A$ be a separable $\mathrm{C}^{*}$-algebra. Let $h \in A$ be a strictly positive element such that $\|h\|=1$. A sequence $\left(h_{n}\right)_{n \in \mathbb{N}}$ is a $h$-spectral approximate unit when there exists a decreasing sequence $\left(\alpha_{n}\right)_{n \in \mathbb{N}}$ in $\left.] 0,1\right]$ converging to 0 such that for each $n \in \mathbb{N}$ we have $h_{n}=\chi_{\left[\alpha_{n}, 1\right]}(h)$, where $\chi_{E}$ is the indicator function of any subset $E$ in $[0,1]$. In particular, by definition, $\left(h_{n}\right)_{n \in \mathbb{N}}$ is a ps-approximate unit of projections in $A^{\prime \prime}$.

Theorem 3.13. Let $A$ be a separable $C^{*}$-algebra, and let $\left(a_{\lambda}\right)_{\lambda \in \Lambda}$ be a bounded net in $A$. Let $h \in A$ be a strictly positive element such that $\|h\|=1$, and $\left(h_{n}\right)_{n \in \mathbb{N}}$ be a h-spectral approximate unit for $A$. Then $\left(a_{\lambda}\right)_{\lambda \in \Lambda}$ converges to 0 in the weaklyuniform topology wu if, and only if it converges to 0 in the inductive topology $\operatorname{ind}\left(h_{n}\right)_{n \in \mathbb{N}}$.

Proof. Since $h$-spectral approximate units are ps-approximate unit, by Lemma (3.8) and Proposition (3.9), the topology $\operatorname{ind}\left(h_{n}\right)_{n \in \mathbb{N}}$ is stronger than wu on any bounded set. We now turn to the converse inclusion.

Let $n \in \mathbb{N}$. Note that $h_{n} \in A^{\prime \prime}$ is a projection. For all $t \in \sigma(h)$ we set $g_{n}(t)=1$ if $t \geq \alpha_{n}$ and $g_{n}(t)=\alpha_{n}^{-1} t$ otherwise, and we set $e_{n}=g_{n}(h) \in C^{*}(h)$. Obviously, $e_{n} h_{n}=h_{n} e_{n}=h_{n}$. We check easily that, since $h_{n}$ is a projection, if $a \in A$ then we have $\left(h_{n} a h_{n}\right)\left(h_{n} e_{n} h_{n}\right)=h_{n} a h_{n} e_{n} h_{n}=h_{n} a h_{n}$ and similarly $\left(h_{n} e_{n} h_{n}\right)\left(h_{n} a h_{n}\right)=h_{n} a h_{n}$, so that $h_{n} \in h_{n} A h_{n}$ and $h_{n}$ is the unit of $h_{n} A h_{n}$ (even though $A$ has no unit in general). Let $\varphi \in S\left(h_{n} A h_{n}\right)$. Define $\iota_{n}(\varphi)(a)=$ $\varphi\left(h_{n} a h_{n}\right)$ for all $a \in A$. The map $\iota_{n}(\varphi)$ is obviously a positive linear functional, since if $a$ is positive then $h_{n} a h_{n}$ is positive as well, and $\|\iota(\varphi)\| \leq 1$. On the other, $\iota_{n}(\varphi)\left(e_{n}\right)=1$, and since $\left\|e_{n}\right\|=1$ we deduce that $\iota_{n}(\varphi)$ is indeed a state 
of $A$. The map $\iota_{n}: S\left(h_{n} A h_{n}\right) \rightarrow S(A)$ is thus an affine map which is obviously continuous when the state spaces are endowed with the weak* topology (it is also immediate that $\iota$ is injective). Let $K_{n}$ be the range of $\iota_{n}$ : by continuity of $\iota_{n}$, the set $K_{n}$ is weak*-compact in $S(A)$.

Let now $\left(a_{\lambda}\right)_{\lambda \in \Lambda}$ be a bounded net in $A$ converging to 0 for wu. For $n \in \mathbb{N}$ and $\lambda \in \Lambda$ we have:

$$
\begin{aligned}
\left\|h_{n} a_{\lambda} h_{n}\right\| & =\sup _{\varphi \in S\left(h_{n} A h_{n}\right)} \varphi\left(h_{n} a_{\lambda} h_{n}\right)=\sup _{\varphi \in S\left(h_{n} A h_{n}\right)} \iota_{n}(\varphi)\left(a_{\lambda}\right) \\
& =\sup _{\varphi \in K_{n}} \varphi\left(a_{\lambda}\right)=p_{K_{n}}\left(a_{\lambda}\right) .
\end{aligned}
$$

Hence, since $a_{\lambda} \underset{\lambda \in \Lambda}{\stackrel{\mathrm{wu}}{\longrightarrow}} 0$ we have $\lim _{\lambda \in \Lambda}\left\|h_{n} a_{\lambda} h_{n}\right\|=\lim _{\lambda \in \Lambda} p_{K_{n}}\left(a_{\lambda}\right)=0$ for all $n \in \mathbb{N}$ so $\left(a_{\lambda}\right)_{\lambda \in \Lambda}$ converges to 0 in the topology $\operatorname{ind}\left(h_{n}\right)_{n \in \mathbb{N}}$. This concludes the proof of our Theorem.

Theorem (3.13) gives us our first insight into a simpler description for the wutopology. It is still a bit difficult to handle a spectral approximate unit, so we shall simplify this result one step further. The following lemma extends mildly on [17, Lemma 3.2.6]:

Lemma 3.14. Let $A$ be a separable $C^{*}$-algebra, $h \in A$ a strictly positive element. Let $\left(a_{\lambda}\right)_{\lambda \in \Lambda}$ be a bounded net in A. Then

$$
\begin{gathered}
\lim _{\lambda \in \Lambda}\left\|h a_{\lambda}\right\|=0 \text { if and only if } \lim _{\lambda \in \Lambda}\left\|b a_{\lambda}\right\|=0 \text { for all } b \in A, \\
\lim _{\lambda \in \Lambda}\left\|a_{\lambda} h\right\|=0 \text { if and only if } \lim _{\lambda \in \Lambda}\left\|a_{\lambda} c\right\|=0 \text { for all } c \in A, \\
\lim _{\lambda \in \Lambda}\left\|h a_{\lambda} h\right\|=0 \text { if and only if } \lim _{\lambda \in \Lambda}\left\|b a_{\lambda} c\right\|=0 \text { for all } b, c \in A .
\end{gathered}
$$

In particular, the strict topology on any bounded subset $h$ of A agree with the topology defined on $h$ by the two seminorms

$$
a \longmapsto\|h a\| \text { and } a \longmapsto\|a h\| \text {. }
$$

Proof. The sufficient conditions above are all trivial, by setting $b=c=h$, and the necessary conditions of (3.3) and (3.4) are found in [17, Lemma 3.2.6], together with the metrizability of the strict topology on bounded subsets of $A$. Now, let $(b, c) \in A$ and assume $\lim _{\lambda \in \Lambda}\left\|h a_{\lambda} h\right\|=0$. In particular, the net $\left(a_{\lambda} h\right)_{\lambda \in \Lambda}$ converges to 0 for the seminorm $a \mapsto\|h a\|$ and thus, by (3.3), we conclude that for all $b \in A$ we have $\lim _{\lambda \in \Lambda}\left\|b a_{\lambda} h\right\|=0$. By (3.4), since the net $\left(b a_{\lambda}\right)_{\lambda \in \Lambda}$ 
converges to 0 for the seminorm $a \mapsto\|a h\|$, we conclude that $\lim _{\lambda \in \Lambda}\left\|b a_{\lambda} c\right\|=0$ as required.

To describe a metric for wu on bounded sets, we need to further our understanding of ps-approximate units. Ps-approximate units are meant to have a similar approximation property in wu as the continuous approximate units have for the strict topology. We shall now establish this, using the following easy lemma as a tool:

Lemma 3.15. Let $X$ be a locally compact Polish space. Let $k$ be a compact subset of $X$. Then the set $K=\left\{\mu \in S\left(C_{0}(X)\right): \mu(k)=1\right\}$ of probability measures supported on $k$ is a weak ${ }^{*}$ compact subset of $S\left(C_{0}(X)\right)$ where $C_{0}(X)$ the is $C^{*}$-algebra of continuous functions on the one-point compactification on $X$ vanishing at infinity, and we identify the continuous linear functionals on $C_{0}(X)$ with the bounded Radon measures on $X$ by the Radon-Riesz theorem.

Proof. Let $\left(\mu_{n}\right)_{n \in \mathbb{N}}$ be a sequence in $K$. The set $K$ is uniformly tight by construction by [6, Theorem 11.5.4., p. 404], so $\left(\mu_{n}\right)_{n \in \mathbb{N}}$ admits a subsequence $\left(\mu_{m(n)}\right)_{n \in \mathbb{N}}$ which converges to $\mu \in S\left(C_{0}(X)\right)$ for the weak* topology. Now, assume that $\mu(k)<1$. Then there exists an open set $U$ in $X \backslash k$ so that $\mu(U)>0$. Hence there exists a nonzero positive compactly supported continuous function $f$ on $U$ and some $\varepsilon>0$ such that $\mu(f)=\varepsilon>0$. We extend $f$ to $X$ by setting $f(X \backslash U)=0$. Now, $f \in C_{0}(X)$ and therefore $\lim _{n \rightarrow \infty} \mu_{m(n)}(f)=\mu(f)=\varepsilon$. Yet, since $f(k)=\{0\}$ by construction, we have $\mu_{n}(f)=0$ for all $n \in \mathbb{N}$, so we reached a contradiction. Hence $\mu(k)=1$ and so $\mu \in K$ which concludes the proof of our lemma, since $X$ is separable so the weak* topology of $S\left(C_{0}(X)\right)$ is metrizable.

We now prove the approximation property of ps-approximate units:

Lemma 3.16. Let $A$ be a separable $C^{*}$-algebra. Let $h \in A$ be a strictly positive element and let $\left(e_{n}\right)_{n \in \mathbb{N}}$ be an h-ps-approximate unit. Then for all $a \in A$, the sequences $\left(a e_{n}\right)_{n \in \mathbb{N}}$ and $\left(e_{n} a\right)_{n \in \mathbb{N}}$ converge to a for the weakly uniform topology wu. Moreover, for all $a \in C^{*}(h)$ :

$$
\lim _{n \rightarrow \infty}\left\|a e_{n}-a\right\|=\lim _{n \rightarrow \infty}\left\|e_{n} a-a\right\|=0 .
$$

Proof. Let $h \in A$ be a strictly positive element such that $\|h\|=1$ and $f_{n}(h)=e_{n}$ where $f_{n}$ is a nondecreasing function on $\sigma(h) \cup\{0\}$ such that $f_{n}(0)=0$, and $\left\|f_{n}\right\| \leq 1$ for all $n \in \mathbb{N}$. Let $a \in A$. Let $K \in \mathfrak{S}$. For any $\varphi \in K$ we have, by Cauchy-Schwarz:

$$
\left|\varphi\left(a e_{n}-a\right)\right|^{2} \leq \varphi\left(a^{*} a\right) \varphi\left(\left(1-e_{n}\right)^{2}\right)
$$


where, as usual, 1 is the unit of $A^{\prime \prime}$. Hence, taking the supremum over $\varphi \in K$ in (3.6) we get $p_{K}\left(a e_{n}-a\right)^{2} \leq\|a\|^{2} p_{K}\left(\left(1-e_{n}\right)^{2}\right)$.

By Lemma (3.8), we have $\lim _{n \rightarrow \infty} p_{K}\left(\left(1-e_{n}\right)^{2}\right)=0$, so we conclude that $p_{K}\left(a e_{n}-a\right) \underset{n \rightarrow \infty}{\longrightarrow} 0$ for all $K \in \mathfrak{S}$. The same reasoning holds for the sequence $\left(e_{n} a\right)_{n \in \mathbb{N}}$.

Now, let $g$ be a continuous function over $\sigma(h) \cup\{0\}$ such that $g(0)=0$. Since $g(0)=0$ and $g$ is continuous on $\sigma(h) \subseteq[0,1]$, there exists $z_{0} \in \sigma(h) \backslash\{0\}$ such that for all $z \in \sigma(h)$ such that $z<z_{0}$ we have $|g(z)| \leq \frac{1}{2} \varepsilon$. Let $k=$ $\left\{z \in \sigma(h): z \geq z_{0}\right\}$. By construction, $k$ is a closed subset of the compact $\sigma(h)$, and we note also $k \subseteq \sigma(h) \backslash\{0\}$. By Lemma (3.15), the set $K=\left\{\mu \in S\left(C_{0}(\sigma(h))\right.\right.$ : $\mu(k)=1\}$ is a weak* compact subset of the state space of $C_{0}(\sigma(h) \backslash\{0\})$. Now, denoting by $\|\cdot\|_{\infty}$ the supremum norm on $L^{\infty}(\sigma(h) \cup\{0\})$, we have:

$$
\left\|f_{n} g-g\right\|_{\infty} \leq \sup _{x \in k}\left\{\left|f_{n}(x) g(x)-g(x)\right|, \varepsilon\right\}
$$

since $\left|f_{n}(x)\right| \leq 1$ by assumption, and since for $x \notin k$ we have $|g(x)| \leq \frac{1}{2} \varepsilon$. Now, we have

$$
\sup _{x \in k}\left|f_{n}(x) g(x)-g(x)\right| \leq\|g\|_{\infty} \sup _{x \in k}\left|f_{n}(x)-1\right| .
$$

The sequence $\left(1-f_{n}\right)_{n \in \mathbb{N}}$ of nonincreasing functions converges on the compact $k$ pointwise, hence uniformly to 0 , as in Lemma (3.8). Therefore, there exists $N \in \mathbb{N}$ such that $\|g\|_{\infty} \sup _{x \in k}\left|f_{n}(x)-1\right| \leq \varepsilon$ for all $n \geq N$. Thus by (3.7) and (3.8) we have for all $n \geq N$ that $\left\|f_{n} g-g\right\|_{\infty} \leq \varepsilon$, so $\lim _{n \rightarrow \infty}\left\|f_{n} g-g\right\|_{\infty}=0$ for all $g \in$ $C_{0}(\sigma(h) \backslash\{0\})$. Consequently for all $a \in C^{*}(h)$ we have $\lim _{n \rightarrow \infty}\left\|f_{n}(h) a-a\right\|=$ 0 .

We can now obtain the main theorem about wu, which provides a simple description of this topology on bounded subsets of $A$ as a metric topology:

Theorem 3.17. Let $A$ be a separable $C^{*}$-algebra. Let $h \in A$ be a strictly positive element. Let $\left(a_{\lambda}\right)_{\lambda \in \Lambda}$ be a bounded net in A. Then $\left(a_{\lambda}\right)_{\lambda \in \Lambda}$ converges to 0 in the weakly-uniform topology wu if, and only if $\lim _{\lambda \in \Lambda}\left\|h a_{\lambda} h\right\|=0$. Equivalently, on any bounded set $B \subseteq A$, the distance $\Delta: a, b \in B \mapsto\|h(b-a) h\|$ metrizes $w u$.

Proof. Let us first assume that the bounded net $\left(a_{\lambda}\right)_{\lambda \in \Lambda}$ converging to 0 in the topology wu. To simplify, assume $\left\|a_{\lambda}\right\| \leq 1$ for all $\lambda \in \Lambda$. Now, let $h \in A$ strictly positive, and assume without loss of generality that $\|h\|=1$. Let $\left(h_{n}\right)_{n \in \mathbb{N}}$ be a $h$-spectral approximate unit. Then, for all $n \in \mathbb{N}$, by Theorem (3.13) we have 
$\lim _{\lambda \in \Lambda}\left\|h_{n} a_{\lambda} h_{n}\right\|=0$. Let $\varepsilon>0$. There exists $N \in \mathbb{N}$ such that, for all $n \geq N$, we have $\left\|h-h h_{n}\right\| \leq \frac{\varepsilon}{2}$ and $\left\|h-h_{n} h\right\| \leq \frac{\varepsilon}{2}$ by Lemma (3.16). We then have:

$$
\begin{aligned}
\left\|h a_{\lambda} h\right\| & \leq\left\|h h_{n} a_{\lambda} h\right\|+\left\|h a_{\lambda} h-h h_{n} a_{\lambda} h\right\| \\
& \leq\|h\|\left\|h_{n} a_{\lambda} h\right\|+\left\|h-h h_{n}\right\|\left\|a_{\lambda} h\right\| \leq\left\|h_{n} a_{\lambda} h\right\|+\frac{\varepsilon}{2} \\
& \leq\left\|h_{n} a_{\lambda} h_{n} h\right\|+\left\|h_{n} a_{\lambda} h-h_{n} a_{\lambda} h_{n} h\right\|+\frac{\varepsilon}{2} \\
& \leq\left\|h_{n} a_{\lambda} h_{n}\right\|\|h\|+\left\|h_{n} a_{\lambda}\right\|\left\|h-h_{n} h\right\|+\frac{\varepsilon}{2} \leq\left\|h_{n} a_{\lambda} h_{n}\right\|+\varepsilon .
\end{aligned}
$$

Hence $0 \leq \overline{\lim }_{\lambda \in \Lambda}\left\|h a_{\lambda} h\right\| \leq \varepsilon$, and since $\varepsilon>0$ is arbitrary, we conclude that $\overline{\lim }_{\lambda \in \Lambda}\left\|h a_{\lambda} h\right\|=0$ and subsequently $\lim _{\lambda \in \Lambda}\left\|h a_{\lambda} h\right\|=0$.

Conversely, we now assume that $\lim _{\lambda \in \Lambda}\left\|h a_{\lambda} h\right\|=0$, with the same notations as above. By the Lemma (3.14), for any elements $b, c \in A$, we have that $\lim _{\lambda \in \Lambda}\left\|b a_{\lambda} c\right\|=0$. This implies that $\left(a_{\lambda}\right)_{\lambda \in \Lambda}$ converges to 0 in ind $\left(e_{n}\right)_{n \in \mathbb{N}}$ for any continuous, Abelian approximate unit $\left(e_{n}\right)_{n \in \mathbb{N}}$ in $A$. This implies wu convergence, as the nets are bounded, by Proposition (3.9). This concludes our theorem.

Corollary 3.18. Let $A$ be a separable $C^{*}$-algebra. The weakly uniform topology restricted to any bounded subsets $A$ is generated by the set of seminorms $(a \in A \longmapsto\|b a c\|)_{b, c \in A}$.

In the next section, we try to return to the state space picture and understand what differs between the strict topology and wu.

\subsection{The Strong Uniform Topology}

We now introduce the topology su, which is modelled after wu, but constructed with the joint continuity of the multiplication on bounded subsets of $A$ in mind as well. The question of the wu-joint continuity of the multiplication on bounded subsets of $A$ is essentially the question of when wu agrees with su on bounded subsets, as we shall see in Theorem (3.20) and (3.21). It turns out that su restricted to bounded subsets of $A$ is the strict topology.

We now introduce our topology su on $A$ :

Definition 3.19. Let $A$ be a separable $\mathrm{C}^{*}$-algebra, and let $\mathfrak{S}$ be the set of all weak* compact subsets of the state space $S(A)$ of $A$. For any $K \in \mathfrak{S}$, we define the seminorms $q_{K}$ by:

$$
q_{K}(a)=\sup _{\varphi \in K}\left(\sqrt[2]{\varphi\left(a^{*} a\right)}, \sqrt[2]{\varphi\left(a a^{*}\right)}\right)
$$


for all $a \in A$. The locally convex topology on $A$ generated by the family $\left(q_{K}\right)_{K \in \mathfrak{S}}$ of seminorms will be called the strongly uniform topology, or su topology.

Using Theorem (3.17) it is immediate that:

Theorem 3.20. Let $A$ be a separable $C^{*}$-algebra. The strict topology and the strong-uniform topology su agree on the bounded subsets of $A$.

Proof. Let $\left(a_{\lambda}\right)_{\lambda \in \Lambda}$ be a bounded net is $A$. Now $\left(a_{\lambda}\right)_{\lambda \in \Lambda} \underset{\lambda \in \Lambda}{\stackrel{\mathrm{su}}{\longrightarrow}} 0$ if, and only if $\left(a_{\lambda}^{*} a_{\lambda}\right)_{\lambda \in \Lambda} \underset{\lambda \in \Lambda}{\stackrel{\mathrm{wu}}{\longrightarrow}} 0$ and $\left(a_{\lambda} a_{\lambda}^{*}\right)_{\lambda \in \Lambda} \underset{\lambda \in \Lambda}{\stackrel{\mathrm{wu}}{\longrightarrow}} 0$ by definition of su. This in turn is equivalent, by Theorem (3.17), to:

$$
0=\lim _{\lambda \in \Lambda}\left\|h a_{\lambda}^{*} a_{\lambda} h\right\|=\lim _{\lambda \in \Lambda}\left\|a_{\lambda} h\right\|^{2} \text { and } 0=\lim _{\lambda \in \Lambda}\left\|h a_{\lambda} a_{\lambda}^{*} h\right\|=\lim _{\lambda \in \Lambda}\left\|h a_{\lambda}\right\|^{2}
$$

which, by Lemma (3.14), is equivalent to the strict convergence of $\left(a_{\lambda}\right)_{\lambda \in \Lambda}$ to 0 .

We can now prove the following necessary and sufficient condition on wu for the multiplication to be wu-jointly continuous:

Theorem 3.21. Let $A$ be a separable $C^{*}$-algebra. The multiplication of $A$ is jointly continuous for the weakly-uniform topology wu on any bounded subset $B$ of $A$ if and only if wu and the strict topology agree on $B$.

Proof. Let $B$ be a bounded subset of $A$. Let $\left(a_{\lambda}\right)_{\lambda \in \Lambda}$ be a net in $B$ converging for the topology wu to $a \in B$. Assume that the multiplication is jointly wucontinuous on $B$. Then the nets $\left(a^{*} a_{\lambda}\right)_{\lambda \in \Lambda},\left(a_{\lambda}^{*} a\right)_{\lambda \in \Lambda}$ and $\left(a_{\lambda}^{*} a_{\lambda}\right)_{\lambda \in \Lambda}$ all converge to $a^{*} a$ for wu, while the nets $\left(a a_{\lambda}^{*}\right)_{\lambda \in \Lambda},\left(a_{\lambda} a^{*}\right)_{\lambda \in \Lambda}$ and $\left(a_{\lambda} a_{\lambda}^{*}\right)_{\lambda \in \Lambda}$ all converge to $a a^{*}$ for wu. We deduce that for any $K \in \mathfrak{S}$, we have $\lim _{\lambda \in \Lambda} \sup _{\varphi \in K} \varphi$ $\left(\left(a_{\lambda}-a\right)^{*}\left(a_{\lambda}-a\right)\right)=0$ and $\lim _{\lambda \in \Lambda} \sup _{\varphi \in K} \varphi\left(\left(a_{\lambda}-a\right)\left(a_{\lambda}-a\right)^{*}\right)=0$. Hence $\left(a_{\lambda}\right)_{\lambda \in \Lambda}$ converges to $a$ for su. By Theorem (3.20), su is the strict topology when restricted to bounded sets, so $\left(a_{\lambda}\right)_{\lambda \in \Lambda}$ converges strictly to $a$ : hence wu is finer than the strict topology on bounded sets. Since by Proposition (3.3), the strict topology is finer than wu, this conclude the necessary condition. The sufficient condition is trivial.

We already encountered in Proposition (3.11) a situation when wu is strictly weaker than the strict topology on a bounded set. We also know that trivially, wu and the strict topology agree (with the norm topology) when $A$ is unital. Another situation when wu and the strict topology agree on bounded sets is when $A$ contains a central, strictly positive element:

Proposition 3.22. Let $A$ be a separable $C^{*}$-algebra with a central strictly positive element. Then the weakly-uniform topology wu and the strict topology agree on bounded subsets of $A$. 
Proof. Let $h \in A$ be a central strictly positive. On any bounded subset $B$ of $A$, the topology wu is generated by the seminorm $a \mapsto\|h a h\|=\left\|h^{2} a\right\|=\left\|a h^{2}\right\|$, and since $h^{2}$ is also strictly positive, this seminorm also generates the strict topology on $B$.

Corollary 3.23. If $A$ is an Abelian separable $C^{*}$-algebra then the strict topology and the wu topology agree on bounded subsets of $A$.

Example 3.24. For a non-Abelian example, consider $A=C_{0}(\mathbb{R}) \otimes M_{2}$, where $M_{2}$ is the algebra of $2 \times 2$ complex matrices. It is neither unital nor Abelian. On the other hand, if $f: x \in \mathbb{R} \longmapsto \exp \left(-x^{2}\right)$ then it is straightforward to check that $f \otimes I_{2}$, where $I_{2}$ is the identity of $M_{2}$, is a strictly positive central element in $A$, hence Proposition (3.22) applies to $A$.

Proposition (3.22) illustrates that, on bounded subsets of $A$, the distinction between wu and the strict topology only appears when, informally, $A$ has a high degree of noncommutativity, and that the nonunital Abelian case may be sometimes misleading in the further study of locally compact quantum metric spaces.

\section{Application to Quasi-Lip-NORMs, Lip-NORMS AND Finite Diameter Quantum Metric Spaces}

We can now rephrase Theorem (2.6) using Theorem (3.17) and Corollary (3.18) to provide a satisfactory answer to Problem (1.1):

Theorem 4.1. Let $A$ be a separable $C^{*}$-algebra. Let $L$ be a seminorm on a dense subspace $\operatorname{dom} L$ of the self-adjoint part $A^{\text {sa }}$ of a $C^{*}$-algebra $A . \quad$ Let $\mathcal{B}_{L}=\{a \in \operatorname{dom} L: L(a) \leq 1,\|a\| \leq 1\}$. Then the following are equivalent:

- The seminorm L is a quasi-Lip-norm (namely L solves Problem (1.1)),

- For all $b, c \in A$, the set $b \mathcal{B}_{L} c$ is norm precompact in $A$,

- There exists a sequence $\left(e_{n}\right)_{n \in \mathbb{N}}$ satisfying Condition (3.5) such that $e_{n} \mathcal{B}_{L} e_{n}$ is norm totally bounded for all $n \in \mathbb{N}$.

- There exists a strictly positive element $h \in A$ such that $h \mathcal{B}_{L} h$ is normprecompact in $A$.

Our setting is particularly satisfactory with finite diameter locally compact quantum metric spaces, since we then get:

Proposition 4.2. Let $A$ be a separable $C^{*}$-algebra. Let $L$ be a quasi-Lip-norm on $A$ and denote by $\kappa_{L}$ the distance induced on the state space $S(A)$ of $A$ by the 
dual of the seminorm $L$. Suppose $\kappa_{L}$ gives $S(A)$ a finite diameter. Then $\kappa_{L}$ agrees with a bounded-Lipschitz metric dual to $L$.

Proof. Since those results are known when $A$ is unital, we assume that $A$ does not have a unit. Let us assume that the Kantorovich distance $\kappa_{L}$ gives $S(A)$ a finite diameter, say $r$. Let $a \in A$ be selfadjoint such that $L(a) \leq 1$. We wish to prove that $\|a\| \leq r$. Since $A$ is not unital, the weak* closure of $S(A)$ is $Q(A)$ and in particular it contains the zero functional. Let $\varepsilon>0$. We can find $\varphi_{\varepsilon}$ such that $\left|\varphi_{\varepsilon}(a)\right| \leq \varepsilon$. Now, since $a$ is self-adjoint:

$$
\|a\|=\sup _{\varphi \in S(A)}|\varphi(a)| \leq \varepsilon+\sup _{\varphi \in S(A)}\left|\varphi(a)-\varphi_{\varepsilon}(a)\right| \leq \varepsilon+\sup _{\varphi \in S(A)} \kappa_{L}\left(\varphi, \varphi_{\varepsilon}\right)
$$

since $L(a) \leq 1$. Hence for all $\varepsilon>0$ we have proven $\|a\| \leq r+\varepsilon$ so $\|a\| \leq r$ as we wished. By definition, the distance $\kappa_{L}$ agrees in this case with $d_{L, r, 1}$.

We conclude with some examples to illustrate Theorem (4.1). Our family of examples is rather simple, but already illustrates that our results are natural:

Example 4.3. Let $(X, \rho)$ be a locally compact separable metric space and $\mathcal{K}$ be the $\mathrm{C}^{*}$-algebra of compact operators. We let $\mathcal{A}_{0}=C_{0}(X, \mathcal{K})$ be the $\mathrm{C}^{*}$-algebra of continuous functions from $X$ into $\mathcal{K}$ and vanishing at infinity. The most immediate choice for a Lipschitz seminorm $l$ on $\mathcal{A}_{0}$ is defined by:

$$
\forall a \in \mathcal{A}_{0} \quad l(a)=\sup _{x, y \in X, x \neq y} \frac{\|a(x)-a(y)\|}{\rho(x, y)} .
$$

Hence, we set $L=\max \{l,\|\|$.$\} as the natural choice for our quasi-Lip-norm on$ $\mathcal{A}_{0}$, and indeed:

Proposition 4.4. Let $(X, \rho)$ be a locally compact separable metric space, and let $l$ be the Lipschitz seminorm defined by $\rho$ on $C_{0}(X)$. The seminorm $L=$ $\max \{l,\|\|$.$\} is a quasi-Lip-norm on C_{0}(X)$, where $\|$.$\| is the supremum norm on$ $C_{0}(X)$.

Proof. We see $\mathcal{K}$ as acting on the separable Hilbert space $\mathcal{H}$ with inner product $\langle.,$.$\rangle and we choose a Hilbert basis \left(e_{n}\right)_{n \in \mathbb{N}}$. For each $n \in \mathbb{N}$ we set $P_{n}$ to be the orthogonal projection on the linear span of $\left\{e_{0}, \ldots, e_{n}\right\}$. Of course, $\left(P_{n}\right)_{n \in \mathbb{N}}$ satisfies Condition (3.5). Moreover $P_{n} \mathcal{A}_{0} P_{n}=C_{0}\left(X, M_{n+1}\right)$ where $M_{n}$ is the $\mathrm{C}^{*_{-}}$ algebra of $n \times n$-complex matrices. Let $f \in \mathcal{A}_{0}$ such that $L(f) \leq 1$ and $f=f^{*}$. Then, for all $k, l \in \mathbb{N}$ :

$$
1 \geq \sup \left\{\frac{\left\langle f(x) e_{k}-f(y) e_{k}, e_{l}\right\rangle}{\rho(x, y)}: x \neq y\right\}
$$


and $\left\|\left\langle f e_{k}, e_{l}\right\rangle\right\|_{C_{0}(X)} \leq 1$, so $\left\langle f e_{k}, e_{l}\right\rangle$ is a 1-Lipschitz map (for the distance $\rho$ ) valued in $[-1,1]$. By Arzéla-Ascoli, this implies that $\left\langle\mathcal{B}_{L} e_{k}, e_{l}\right\rangle$ is norm totally bounded in $C_{0}(X)$. Hence $P_{n} \mathcal{B}_{L} P_{n}$ is norm totally bounded in $C_{0}\left(X, M_{n+1}\right)$ (by a diagonal argument). Therefore $\mathcal{B}_{L}$ is wu-totally bounded.

The construction of $L$ reflects the idea that $C_{0}(X)$ and $\mathcal{A}_{0}$ should have the same geometry (Morita equivalence). In particular, if $\mathbb{Z}$ acts properly and freely on a locally compact space $Y$ so that the orbit space is $X$ then $\mathbb{Z} \ltimes C_{0}(Y)$ is Morita equivalent to $\mathcal{A}_{0}$. Moreover, if $H_{3}(X, \mathbb{Z})=0$ then $\mathbb{Z} \ltimes C_{0}(Y)$ is $\mathcal{A}_{0}$ by [4, Corollary 10.9.6]. If $d$ is a metric on $Y$ then one can define $\rho$ on $X$ by $\rho(\mathcal{O}(x), \mathcal{O}(y))=\inf \left\{d\left(z \cdot x, z^{\prime} \cdot y\right): z, z^{\prime} \in \mathbb{Z}\right\}$ (where $\mathcal{O}(x)$ is the orbit of $x \in Y)$, so that such crossed-products fit in this example.

Example (4.3), though rather simple, contains in fact an important lesson: indeed, let us use the notations of the proof of Proposition (3.11). The sequence $\left(S_{n}^{*}\right)_{n \in \mathbb{N}}$ converges to 0 for wu, though it is immediate that $\left(S_{n}^{*}\right)_{n \in \mathbb{N}}$ does not have any subsequence which converges (to 0 ) in the strict topology. First, simply by setting $X=\{1\}$ in Example (4.3), we observe that $L\left(S_{n}\right)=\left\|S_{n}\right\|=1$ and this is counterexample to the trivial fact that $\{a \in \mathcal{K}:\|a\| \leq 1\}$ is not strictly totally bounded, though it is wu totally bounded. On the other hand, if $(X, \rho)$ is an arbitrary separable locally compact metric space, we can pick $f \in C_{0}(X)$ such that $\|f\| \leq 1$ and which is 1-Lipschitz for $\rho$. We then define $a_{n} \in C_{0}(X, \mathcal{K})$ by $a_{n}=f \otimes S_{n}^{*}$. It is easy to check that $L\left(a_{n}\right) \leq 1$ and $\left\|a_{n}\right\| \leq 1$ for all $n \in \mathbb{N}$, yet again $\left(a_{n}\right)_{n \in \mathbb{N}}$ has not strictly convergent subsequence. Thus, $\{a \in \mathcal{A}: L(a) \leq 1$ and $\|a\| \leq 1\}$ is wu-totally bounded yet not strictly totally bounded, even for such a natural construction of a Lipschitz norm.

\section{REFERENCES}

1. 1. E. M. Alfsen, Compact convex sets and boundary integrals, Ergebnisse Math., Vol. 57, Springer-Verlag, 1971.

2. A. Connes, Compact metric spaces, fredholm modules and hyperfiniteness, Ergodic Theory and Dynamical Systems, 9(2) (1989), 207-220.

3. A. Connes, Noncommutative geometry, Academic Press, San Diego, 1994.

4. J. Dixmier, Les $C^{*}$-Algebres et leur repr'esentations, Gauthier-Villars, 1969, (reprint) Editions Jacques Gabay, 1996.

5. R S. Doran and J. Wichmann, Approximate identities and factorization in banach modules, Lecture Notes in Mathematics, Vol. 768, Springer-Verlag, 1979.

6. R. M. Dudley, Real analysis and probability, Cambridge Studies in Advanced Mathematics, Vol. 74, 2002 ed., Cambridge University Press, 2002. 
7. M. Gromov, Metric structures for Riemannian and non-Riemannian spaces, Progress in Mathematics, Birkhäuser, 1999.

8. R. V. Kadison, A representation theory for commutative topological algebras, Mem. Amer. Math. Soc., 7 (1951).

9. F. Latremoliere, Approximation of the quantum tori by finite quantum tori for the quantum gromov-hausdorff distance, J. Funct. Anal., 223 (2005), 365-395.

10. G. K. Pedersen, $C *$-Algebras and their automorphism groups, Academic Press, 1979.

11. M. A. Rieffel, Metrics on states from actions of compact groups, Documenta Mathematica, 3 (1998), 215-229.

12. — Metrics on state spaces, Documenta Math., 4 (1999), 559-600.

13. - - Matrix algebras converge to the sphere for quantum Gromov-VHausdorff distance, Mem. Amer. Math. Soc., (2001),

14. — Gromov-Hausdorff distance for quantum metric spaces, Mem. Amer. Math. Soc., 168 (March 2004), no. 796,

15. W. Rudin, Functional analysis, 2nd edition, McGraw-Hill, 1991.

16. N. Weaver, Lipschitz algebras, World Scientific, 1999.

17. N. E. Wegge Olsen, $K$-theory and $C *$-Algebras, Oxford Science Publications, Oxford University Press, 1992.

Frédéric Latrémoliere

Department of Mathematics,

University of Toronto,

Canada

E-mail: frederic@math.toronto.edu

http://www.math.toronto.edu/frederic 